Available online at http://jnfa.mathres.org
MATHRES Journal of Nonlinear Functional Analysis
A

\title{
BOUNDED PERTURBATION RESILIENCE OF A VISCOSITY ITERATIVE METHOD FOR SPLIT FEASIBILITY PROBLEMS
}

\author{
PEICHAO DUAN*, XUBANG ZHENG \\ College of Science, Civil Aviation University of China, Tianjin 300300, China
}

\begin{abstract}
In this article, we propose a viscosity approximation method to solve a split feasibility problem. The bounded perturbation resilience of the method is investigated in Hilbert spaces. As tools, averaged mappings and resolvents of maximal monotone operators are technically maneuvered to facilitate the proofs of the main results. Under mild conditions, we prove that our algorithms strongly converge to a solution of the split feasibility problem, which is also the unique solution of a variational inequality problem. Furthermore, we also show the convergence and effectiveness of the algorithms by a numerical example. Keywords. Split feasibility problem; Maximal monotone operator; Averaged mapping; Variational inequality problem.
\end{abstract}

2010 Mathematics Subject Classification. 47H05, 47H09, 47H10, 47J25.

\section{INTRODUCTION}

Let $H_{1}$ and $H_{2}$ be two real Hilbert spaces with inner product $\langle$,$\rangle and induced norm \|\cdot\|$. Let $C$ be a nonempty closed and convex subset of $H_{1}$ and let $P_{C}$ the metric projection from $H_{1}$ onto $C$. Let $Q$ be a nonempty closed convex subsets of $H_{2}$ and let $P_{Q}$ be the metric projection from $H_{2}$ onto $Q$. Recall that the split feasibility problem (SFP) is to find a point $x^{*}$ satisfying the conditions

$$
x^{*} \in C \text { and } A x^{*} \in Q,
$$

where $A$ is a bounded linear operator from $H_{1}$ to $H_{2}$. Assume that $C \cap A^{-1} Q$ is nonempty (i.e., problem (1.1) has a solution). It is not hard to see that $x^{*} \in C \cap A^{-1} Q$ is equivalent to

$$
x^{*}=P_{C}\left(I-\lambda A^{*}\left(I-P_{Q}\right) A\right) x^{*}
$$

where $\lambda>0$ is some positive real number and $A^{*}$ is the adjoint operator of $A$.

In 1994, the SFP was first introduced by Censor and Elfving [1]. They used their algorithm to solve the SFP in finite-dimensional Euclidean spaces. In 2002, Byrne [2] improved Censor and Elfving's

${ }^{*}$ Corresponding author.

E-mail addresses: pcduancauc@126.com (P. Duan), xbzheng224@163.com (X. Zheng).

Received September 19, 2018; Accepted December 19, 2018.

(C)2019 Journal of Nonlinear Functional Analysis 
algorithm in infinite-dimensional spaces and presented a new method called $C Q$ algorithm for solving SFP (1.1):

$$
x_{n+1}=P_{C}\left(I-\lambda A^{*}\left(I-P_{Q}\right) A\right) x_{n},
$$

In 2007, Censor, Motova and Segal [3] studied a multiple-sets split-feasibility problem. In 2010, Moudafi [4] proposed an iterative method to solve split common fixed point problems of quasi-nonexpansive mappings. In 2014, combining the Moudafi's method with the Halpern iterative method, Kraikaew and Saejung [5] proposed a new iterative algorithm that does not involve projection operators to solve a split common fixed point problem (SCFP). More precisely, they gave the following algorithm:

$$
\left\{\begin{array}{l}
x_{0} \in H_{1}, \\
x_{n+1}=\alpha_{n} x_{0}+\left(1-\alpha_{n}\right) U\left(x_{n}+\gamma A^{*}(T-I) A x_{n}\right), \quad n \geq 0 .
\end{array}\right.
$$

Under the reasonable conditions, they proved that $\left\{x_{n}\right\}$ is strongly convergent. If we take $U$ and $T$ as the projection operators $P_{C}$ and $P_{Q}$, respectively, then Fix $(U)=C$ and $F i x(T)=Q$. Hence the SCFP immediately reduces to the SFP and the above algorithm can solve the SFP. In 2015, Takahashi, Xu and Yao [6] proposed the following algorithm:

$$
x_{n+1}=J_{\lambda_{n}}^{B}\left(x_{n}-\tau_{n} A^{*}(I-T) A x_{n}\right),
$$

$J_{\lambda_{n}}^{B}$ is the resolvent operator of maximal monotone operator $B$ and $T$ is a nonexpansive mapping on $H_{2}$. They proved that the sequence generated by the above iterative process converges weakly to a point $x^{*} \in B^{-1} 0 \cap A^{-1} F i x(T)$ in the framework of Hilbert spaces. We also describe that this problem is to find a point $x^{*} \in H_{1}$ such that

$$
0 \in B x^{*} \text { and } A x^{*} \in \operatorname{Fix}(T) .
$$

Especially, if $B=\partial I_{C}=N_{C}$ and $T=P_{Q}$, where

$$
I_{C} x=\left\{\begin{array}{l}
0, x \in C \\
\infty, x \bar{\in} C
\end{array}\right.
$$

is the indicator function of closed convex subset $C$ and

$$
N_{C} x=\{u \in H:\langle u, x-y\rangle \geq 0, \forall y \in C\}
$$

is the normal cone to $C$ at $x \in C$. Then problem (1.5) reduces to SFP (1.1). We denote the solution set of problem (1.5) by $S=B^{-1} 0 \cap A^{-1} F i x(T)$.

The following lemma is very useful in constructing iterative algorithms.

Lemma 1.1. [6] Let $H_{1}$ and $H_{2}$ be Hilbert spaces. Let $B: H_{1} \rightarrow 2^{H_{1}}$ be a maximal monotone mapping and let $J_{\lambda}^{B}=(I+\lambda B)^{-1}$ be the resolvent of $B$ for $\lambda>0$. Let $T: H_{2} \rightarrow H_{2}$ be a nonexpansive mapping and let $A: H_{1} \rightarrow H_{2}$ be a bounded linear operator. Suppose that $B^{-1} 0 \cap A^{-1} F i x(T) \neq \emptyset$. Let $\lambda, \tau>0$. Then the following equality holds:

$$
\operatorname{Fix}\left(J_{\lambda}^{B}\left(I-\tau A^{*}(I-T) A\right)\right)=\left(A^{*}(I-T) A+B\right)^{-1} 0=B^{-1} 0 \cap A^{-1} F i x(T) .
$$

For the SFP and its extensions, many authors have studied them via fixed-point methods and weakstrong convergence theorems of solutions have been established in Hilbert or Banach spaces; see [7, $8,9,10,11,12,13,14,15]$ and the references therein. Recently, bounded perturbation resilience of iterative methods have also been extensively studied; see $[16,17,18,19,20]$ and the references therein. 
This problem received much attention due to its applications in convex feasibility problems [21], inverse problems of radiation therapy [22] and image reconstruction [23], and so on.

Let $\mathbf{P}$ denote an algorithm operator, if the iteration $x_{n+1}=\mathbf{P} x_{n}$ is replaced by $x_{n+1}=\mathbf{P}\left(x_{n}+\beta_{n} v_{n}\right)$, where $\beta_{n}$ is a sequence of nonnegative real numbers $v_{n}$ is a sequence in $H$ such that

$$
\sum_{n=0}^{\infty} \beta_{n}<\infty \text { and }\left\|v_{n}\right\| \leq M
$$

If the algorithm is still convergent, then algorithm $\mathbf{P}$ is bounded perturbation resilient; see [16] and the references therein.

In 2016, Jin, Censor and Jiang [19] introduced the projected scaled gradient (PSG) method with bounded perturbations for solving the following minimization problem:

$$
\min _{x \in C} f(x)
$$

where $f$ is a continuous differentiable, convex function. Their method generates a sequence $\left\{x_{n}\right\}$ by the iterative scheme:

$$
x_{n+1}=P_{C}\left(x_{n}-\gamma_{n} D\left(x_{n}\right) \nabla f\left(x_{n}\right)+e\left(x_{n}\right)\right), n \geq 0,
$$

where $D\left(x_{n}\right)$ is a diagonal scaling matrix. Under suitable conditions, they obtained a convergence theorem.

Recently, Xu [20] projected the superiorization techniques for the relaxed PSG. The iterative algorithm is defined as following:

$$
x_{n+1}=\left(1-\tau_{n}\right) x_{n}+\tau_{n} P_{C}\left(x_{n}-\gamma_{n} D\left(x_{n}\right) \nabla f\left(x_{n}\right)+e\left(x_{n}\right)\right), n \geq 0,
$$

where $\left\{\tau_{n}\right\}$ is a sequence in $[0,1]$. The weak convergence was proved in [20].

Very recently, Guo and Cui [24] presented the following modified proximal gradient algorithm with perturbations for solving non-smooth composite convex optimization problem $\min _{x \in H}(f(x)+g(x))$ :

$$
x_{n+1}=\alpha_{n} h\left(x_{n}\right)+\left(1-\alpha_{n}\right) \operatorname{prox}_{\lambda_{n} g}\left(x_{n}-\lambda_{n} \nabla f\left(x_{n}\right)+e\left(x_{n}\right)\right), \quad n \geq 0,
$$

where $h$ is contractive. They obtained strong convergence and bounded resilience of the above method.

In this paper, motivated by the results in $[6,16,20,24]$, we propose a viscosity approximation method for solving problem (1.5) and prove our iterative method is bounded perturbation resilient. We prove the convergence point of the iterative method which is also the unique solution of some variational inequality problem. a numerical example is also given to demonstrate the effectiveness of our iterative schemes. Our method for problem (1.5) is as follows:

$$
x_{n+1}=\alpha_{n} h\left(x_{n}\right)+\left(1-\alpha_{n}\right) J_{\lambda_{n}}^{B}\left(x_{n}-\tau_{n} A^{*}(I-T) A x_{n}+e\left(x_{n}\right)\right) .
$$

We give the bounded perturbation of (1.12) yields a sequence $\left\{x_{n}\right\}$ generated by the iterative process:

$$
\left\{\begin{array}{l}
y_{n}=x_{n}+\beta_{n} v_{n}, \\
x_{n+1}=\alpha_{n} h\left(y_{n}\right)+\left(1-\alpha_{n}\right) J_{\lambda_{n}}^{B}\left(y_{n}-\tau_{n} A^{*}(I-T) A y_{n}+e\left(y_{n}\right)\right),
\end{array}\right.
$$

We also discuss the convergence of the viscosity method and show it is bounded perturbation resilient. 


\section{PRELIMINARIES}

Let $\left\{x_{n}\right\}$ be a sequence in a real Hilbert space $H$. We adopt the following notations:

(1) Denote $\left\{x_{n}\right\}$ converging weakly to $x$ by $x_{n} \rightarrow x$ and $\left\{x_{n}\right\}$ converging strongly to $x$ by $x_{n} \rightarrow x$.

(2) Use $\operatorname{Fix}(T)$ to denote the set of fixed points of mapping $T$; that is, $\operatorname{Fix}(T)=\{x \in H: T x=x\}$.

(3) Denote the weak $\omega$-limit set of $\left\{x_{n}\right\}$ by $\omega_{w}\left(x_{n}\right):=\left\{x: \exists x_{n_{j}} \rightarrow x\right\}$.

We also need the following definitions.

Definition 2.1. A mapping $F: H \rightarrow H$ is said to be

(i) Lipschizian if there exists a positive constant $L$ such that

$$
\|F x-F y\| \leq L\|x-y\|, \quad \forall x, y \in H .
$$

In particular, if $L=1$, we say that $F$ is nonexpansive, namely,

$$
\|F x-F y\| \leq\|x-y\|, \forall x, y \in H
$$

if $L \in[0,1)$, we say that $F$ is contractive.

(ii) $\alpha$-averaged mapping ( $\alpha$-av for short) if

$$
F=(1-\alpha) I+\alpha T,
$$

where $\alpha \in[0,1)$ and $T: H \rightarrow H$ is nonexpansive.

Definition 2.2. A mapping $B: H \rightarrow H$ is said to be

(i) monotone if

$$
\langle B x-B y, x-y\rangle \geq 0, \forall x, y \in H .
$$

(ii) $\eta$-strongly monotone if there exists a positive constant $\eta$ such that

$$
\langle B x-B y, x-y\rangle \geq \eta\|x-y\|^{2}, \forall x, y \in H .
$$

(iii) $\alpha$-inverse strongly monotone(for short $\alpha$-ism) if there exists a positive constant $\alpha$ such that

$$
\langle B x-B y, x-y\rangle \geq \alpha\|B x-B y\|^{2}, \quad \forall x, y \in H .
$$

In particular, if $\alpha=1$, we say that $B$ is firmly nonexpansive, namely,

$$
\langle B x-B y, x-y\rangle \geq\|B x-B y\|^{2}, \forall x, y \in H .
$$

Definition 2.3. Let $B: H \rightarrow H$ be a monotone mapping. Then $B$ is maximal monotone if there exists no monotone operator $A: H \rightarrow 2^{H}$ such that graA properly contains graB, i.e., for every $(x, u) \in H \times H$,

$$
(x, u) \in \operatorname{graB} \Leftrightarrow \forall(y, v) \in \operatorname{graB},\langle x-y, u-v\rangle \geq 0 .
$$

The following two lemmas are trivial.

Lemma 2.4. Let $H$ be a real Hilbert space. There holds the following inequality

$$
\|x+y\|^{2} \leq\|x\|^{2}+2\langle x+y, y\rangle, \forall x, y \in H .
$$

Lemma 2.5. Let $h: H \rightarrow H$ be a $\rho$-contraction with $\rho \in(0,1)$ and let $T: H \rightarrow H$ be a nonexpansive mapping. Then 
(i) $I-h$ is $(1-\rho)$-strongly monotone:

$$
\langle(I-h) x-(I-h) y, x-y\rangle \geq(1-\rho)\|x-y\|^{2}, \quad \forall x, y \in H .
$$

(ii) $I-T$ is monotone:

$$
\langle(I-T) x-(I-T) y, x-y\rangle \geq 0, \quad \forall x, y \in H .
$$

Proposition 2.6. [25]

(i) If $T_{1}, T_{2}, \cdots, T_{n}$ are averaged mappings, then $T_{n} T_{n-1} \cdots T_{1}$ is averaged. In particular, if $T_{i}$ is $\alpha_{i}$-av, $i=1,2$, where $\alpha_{i} \in(0,1)$, then $T_{2} T_{1}$ is $\left(\alpha_{2}+\alpha_{1}-\alpha_{2} \alpha_{1}\right)$-av.

(ii) If $\left\{T_{i}\right\}_{i=1}^{N}$ are averaged and have a common fixed point, then

$$
\bigcap_{i=1}^{N} \operatorname{Fix}\left(T_{i}\right)=\operatorname{Fix}\left(T_{1} \ldots T_{N}\right) .
$$

(iii) A mapping $T$ is nonexpansive if and only if $I-T$ is $\frac{1}{2}-i s m$.

(iv) If $T$ is $v$-ism, then, for $\tau>0, \tau T$ is $\frac{v}{\tau}$-ism.

(v) $T$ is averaged if and only if $I-T$ is $v$-ism for some $v>\frac{1}{2}$. Indeed, for $0<\alpha<1, T$ is $\alpha$-averaged if and only if $I-T$ is $\frac{1}{2 \alpha}$-ism.

Proposition 2.7. [6] Assume that $H_{1}$ and $H_{2}$ are Hilbert spaces. Let $B: H_{1} \rightarrow 2^{H_{1}}$ be a maximal monotone mapping and let $A: H_{1} \rightarrow H_{2}$ be a bounded linear operator such that $A \neq 0$. Let $T: H_{2} \rightarrow H_{2}$ be a nonexpansive mapping. Then

(i) $A^{*}(I-T) A$ is $\frac{1}{2\|A\|^{2}}$ ism.

(ii) For $0<\tau<\frac{1}{\|A\|^{2}}$,

$$
I-\tau A^{*}(I-T) A \text { is } \tau\|A\|^{2} \text {-averaged and } J_{\lambda}^{B}\left(I-\tau A^{*}(I-T) A\right) \text { is } \frac{1+\tau\|A\|^{2}}{2} \text {-averaged. }
$$

Lemma 2.8. [26] Let $H$ be a real Hilbert space, and let $T: H \rightarrow H$ be a nonexpansive mapping with $\operatorname{Fix}(T) \neq \emptyset$. If $\left\{x_{n}\right\}$ is a sequence in $H$ weakly converging to $x$ and if $\left\{(I-T) x_{n}\right\}$ converges strongly to $y$, then $(I-T) x=y$; in particulary, if $y=0$, then $x \in F i x(T)$.

Lemma 2.9. [27] Assume $\left\{s_{n}\right\}$ is a sequence of nonnegative real numbers such that

$$
\begin{gathered}
s_{n+1} \leq\left(1-\gamma_{n}\right) s_{n}+\gamma_{n} \delta_{n}, n \geq 0, \\
s_{n+1} \leq s_{n}-\eta_{n}+\varphi_{n}, \quad n \geq 0,
\end{gathered}
$$

where $\left\{\gamma_{n}\right\}$ is a sequence in $(0,1),\left\{\eta_{n}\right\}$ is a sequence of nonnegative real numbers and $\left\{\delta_{n}\right\}$ and $\left\{\varphi_{n}\right\}$ are two sequences in $\mathbb{R}$ such that

(i) $\sum_{n=0}^{\infty} \gamma_{n}=\infty$,

(ii) $\lim _{n \rightarrow \infty} \varphi_{n}=0$,

(iii) $\lim _{k \rightarrow \infty} \eta_{n_{k}}=0$ implies limsup $\operatorname{sim}_{k \rightarrow \infty} \delta_{n_{k}} \leq 0$ for any subsequence $\left(n_{k}\right) \subset(n)$.

Then $\lim _{n \rightarrow \infty} s_{n}=0$.

Lemma 2.10. [28] Let $B$ be a maximal monotone operator. Let $J_{\lambda}^{B}=(I+\lambda B)^{-1}$ and $J_{\mu}^{B}=(I+\mu B)^{-1}$, where $\lambda>0$ and $\mu>0$ are two real numbers, be the resolvent operators of $B$. Then

$$
J_{\lambda}^{B} x=J_{\mu}^{B}\left(\frac{\mu}{\lambda} x+\left(1-\frac{\mu}{\lambda}\right) J_{\lambda}^{B} x\right), \quad \forall x \in H .
$$




\section{MAIN RESULTS}

Let $C$ be a nonempty closed and convex subset of a Hilbert space $H$. Let $N: C \rightarrow C$ be a nonexpansive mapping and let $h: H \rightarrow H$ be a $\rho$-contractive mapping. In 2000, Moudafi [29] proposed the following viscosity approximation method:

$$
x_{n+1}=\alpha_{n} h\left(x_{n}\right)+\left(1-\alpha_{n}\right) N x_{n},
$$

where $\left\{\alpha_{n}\right\}$ is some sequence in $(0,1)$. He proved that the viscosity algorithm converges strongly to a fixed point $x^{*}$ of nonexpansive mapping $N$ and $x^{*}$ also uniquely solves the following variational inequality

$$
\left\langle(I-h) x^{*}, \tilde{x}-x^{*}\right\rangle \geq 0, \quad \forall \tilde{x} \in F i x(N) .
$$

Recently, some authors extended the above results to the framework of Banach spaces; see [30, 31, 32] and the references therein.

In this section, we present a viscosity iterative algorithm for solving problem (1.5). Rewrite iteration (1.12) as

$$
\begin{aligned}
x_{n+1} & =\alpha_{n} h\left(x_{n}\right)+\left(1-\alpha_{n}\right) J_{\lambda_{n}}^{B}\left(x_{n}-\tau_{n} A^{*}(I-T) A x_{n}+e\left(x_{n}\right)\right) \\
& =\alpha_{n} h\left(x_{n}\right)+\left(1-\alpha_{n}\right)\left(J_{\lambda_{n}}^{B}\left(x_{n}-\tau_{n} A^{*}(I-T) A x_{n}\right)+\tilde{e}_{n}\right),
\end{aligned}
$$

Since $J_{\lambda_{n}}^{B}$ is nonexpansive, we find that

$$
\begin{aligned}
\left\|\tilde{e}_{n}\right\| & =\left\|J_{\lambda_{n}}^{B}\left(x_{n}-\tau_{n} A^{*}(I-T) A x_{n}+e\left(x_{n}\right)\right)-J_{\lambda_{n}}^{B}\left(x_{n}-\tau_{n} A^{*}(I-T) A x_{n}\right)\right\| \\
& \leq\left\|e\left(x_{n}\right)\right\| .
\end{aligned}
$$

Theorem 3.1. Let $H_{1}, H_{2}$ be two real Hilbert spaces and let $A: H_{1} \rightarrow H_{2}$ be a bounded linear operator with $L=\left\|A^{*} A\right\|$, where $A^{*}$ is the adjoint of $A$. Suppose that $B: H_{1} \rightarrow 2^{H_{1}}$ is a maximal monotone operator and $\mathrm{T}: \mathrm{H}_{2} \rightarrow \mathrm{H}_{2}$ is a nonexpansive mapping. Assume that $S=B^{-1} 0 \cap A^{-1} \mathrm{Fix}(T) \neq \emptyset$. Let $h$ be a $\rho$ contractive on $H_{1}$ with $0 \leq \rho<1$. Choose $x_{0} \in H_{1}$ arbitrarily and define a sequence $\left\{x_{n}\right\}$ in the following manner:

$$
x_{n+1}=\alpha_{n} h\left(x_{n}\right)+\left(1-\alpha_{n}\right) J_{\lambda_{n}}^{B}\left(x_{n}-\tau_{n} A^{*}(I-T) A x_{n}+e\left(x_{n}\right)\right) .
$$

If the following conditions are satisfied:

(i) $\lim _{n \rightarrow \infty} \alpha_{n}=0$ and $\sum_{n=0}^{\infty} \alpha_{n}=\infty$;

(ii) $0<\liminf _{n \rightarrow \infty} \lambda_{n} \leq \limsup _{n \rightarrow \infty} \lambda_{n}<\infty$;

(iii) $0<\liminf _{n \rightarrow \infty} \tau_{n} \leq \limsup _{n \rightarrow \infty} \tau_{n}<\frac{1}{L}$;

(iv) $\sum_{n=0}^{\infty}\left\|e\left(x_{n}\right)\right\|<\infty$,

then $\left\{x_{n}\right\}$ converges strongly to $x^{*} \in B^{-1} 0 \cap A^{-1} F i x(T)$, which is also the unique solution of variational inequality problem (3.1).

Proof. Set $V_{n}:=J_{\lambda_{n}}^{B}\left(I-\tau_{n} A^{*}(I-T) A\right)$. From Proposition 2.7, it is easy to obtain that $J_{\lambda_{n}}^{B}\left(I-\tau_{n} A^{*}(I-\right.$ $T) A)$ is $\frac{1+\tau_{n} L}{2}$-av as $0<\tau_{n}<1 / L$.

Next, we split the proof into 3 steps.

Step 1. Show that $\left\{x_{n}\right\}$ is bounded. 
For any $z \in S$, we have

$$
\begin{aligned}
\left\|x_{n+1}-z\right\| & =\left\|\alpha_{n} h\left(x_{n}\right)+\left(1-\alpha_{n}\right)\left(V_{n} x_{n}+\tilde{e}_{n}\right)-z\right\| \\
& =\left\|\alpha_{n}\left(h\left(x_{n}\right)-z\right)+\left(1-\alpha_{n}\right)\left(V_{n} x_{n}-z\right)+\left(1-\alpha_{n}\right) \tilde{e}_{n}\right\| \\
& \leq \alpha_{n}\left\|h\left(x_{n}\right)-h(z)\right\|+\alpha_{n}\|h(z)-z\|+\left(1-\alpha_{n}\right)\left\|V_{n} x_{n}-z\right\|+\left\|\tilde{e}_{n}\right\| \\
& \leq \alpha_{n} \rho\left\|x_{n}-z\right\|+\alpha_{n}\|h(z)-z\|+\left(1-\alpha_{n}\right)\left\|x_{n}-z\right\|+\left\|\tilde{e}_{n}\right\| \\
& =\left(1-\alpha_{n}(1-\rho)\right)\left\|x_{n}-z\right\|+\alpha_{n}(1-\rho) \frac{\|h(z)-z\|+\left\|\tilde{e}_{n}\right\| / \alpha_{n}}{1-\rho} .
\end{aligned}
$$

From conditions (i), (iv) and $\alpha_{n}>0$, we find that $\left\{\left\|\tilde{e}_{n}\right\| / \alpha_{n}\right\}$ is bounded. Thus there exists some $M_{1}>0$ such that $\sup \left\{\|h(z)-z\|+\left\|\tilde{e}_{n}\right\| / \alpha_{n}\right\} \leq M_{1}$ for all $n \geq 0$. An induction argument shows that

$$
\left\|x_{n}-z\right\| \leq \max \left\{\left\|x_{0}-z\right\|, \frac{M_{1}}{1-\rho}\right\},
$$

which implies that sequence $\left\{x_{n}\right\}$ is bounded, so are $\left\{h\left(x_{n}\right)\right\},\left\{V_{n} x_{n}\right\}$ and $\left\{A^{*}(I-T) A x_{n}\right\}$.

Step 2. Show that for any sequence $\left(n_{k}\right) \subset(n), \lim _{k \rightarrow \infty}\left\|x_{n_{k}}-V_{n_{k}} x_{n_{k}}\right\|=0$.

Fixing $z \in S$, we have

$$
\begin{aligned}
& \left\|x_{n+1}-z\right\|^{2} \\
& =\left\|\alpha_{n} h\left(x_{n}\right)+\left(1-\alpha_{n}\right)\left(V_{n} x_{n}+\tilde{e}_{n}\right)-z\right\|^{2} \\
& \leq\left\|\alpha_{n} h\left(x_{n}\right)+\left(1-\alpha_{n}\right) V_{n} x_{n}-z\right\|^{2}+2\left\langle\alpha_{n} h\left(x_{n}\right)+\left(1-\alpha_{n}\right) V_{n} x_{n}-z,\left(1-\alpha_{n}\right) \tilde{e}_{n}\right\rangle+\left\|\tilde{e}_{n}\right\|^{2} \\
& \leq \alpha_{n}^{2}\left\|h\left(x_{n}\right)-z\right\|^{2}+\left(1-\alpha_{n}\right)^{2}\left\|V_{n} x_{n}-z\right\|^{2}+2 \alpha_{n}\left(1-\alpha_{n}\right)\left\langle h\left(x_{n}\right)-z, V_{n} x_{n}-z\right\rangle \\
& \quad+\left(2 \alpha_{n}\left\|h\left(x_{n}\right)-z\right\|+2\left(1-\alpha_{n}\right)\left\|x_{n}-z\right\|+\left\|\tilde{e}_{n}\right\|\right)\left\|\tilde{e}_{n}\right\| \\
& \leq 2 \alpha_{n}^{2}\left(\left\|h\left(x_{n}\right)-h(z)\right\|^{2}+\|h(z)-z\|^{2}\right)+\left(1-\alpha_{n}\right)^{2}\left\|V_{n} x_{n}-z\right\|^{2} \\
& \quad+2 \alpha_{n}\left(1-\alpha_{n}\right)\left\langle h\left(x_{n}\right)-z, V_{n} x_{n}-z\right\rangle+M_{2}\left\|\tilde{e}_{n}\right\| \\
& \leq 2 \alpha_{n}^{2}\left(\left\|h\left(x_{n}\right)-h(z)\right\|^{2}+\|h(z)-z\|^{2}\right)+\left(1-\alpha_{n}\right)^{2}\left\|V_{n} x_{n}-z\right\|^{2} \\
& \quad+2 \alpha_{n}\left(1-\alpha_{n}\right)\left(\left\|h\left(x_{n}\right)-h(z)\right\|\left\|x_{n}-z\right\|+\left\langle h(z)-z, V_{n} x_{n}-z\right\rangle\right)+M_{2}\left\|\tilde{e}_{n}\right\| \\
& \leq\left(1-\alpha_{n}\left(2-\alpha_{n}\left(1+2 \rho^{2}\right)-2\left(1-\alpha_{n}\right) \rho\right)\right)\left\|x_{n}-z\right\|^{2}+2 \alpha_{n}\left(1-\alpha_{n}\right)\left\langle h(z)-z, V_{n} x_{n}-z\right\rangle \\
& \quad+2 \alpha_{n}^{2}\|h(z)-z\|^{2}+M_{2}\left\|\tilde{e}_{n}\right\|,
\end{aligned}
$$

where

$$
M_{2}:=\sup _{n \in \mathbb{N}}\left\{2 \alpha_{n}\left\|h\left(x_{n}\right)-z\right\|+2\left(1-\alpha_{n}\right)\left\|x_{n}-z\right\|+\left\|\tilde{e}_{n}\right\|\right\}
$$

Note that

$$
V_{n}=J_{\lambda_{n}}^{B}\left(I-\tau_{n} A^{*}(I-T) A\right)=\left(1-w_{n}\right) I+w_{n} U_{n},
$$

where $w_{n}=\frac{1+\tau_{n} L}{2}$, and $U_{n}$ is nonexpansive. By condition (iii), we get that

$$
\frac{1}{2}<\liminf _{n \rightarrow \infty} w_{n} \leq \limsup _{n \rightarrow \infty} w_{n}<1
$$


Since $z \in S$, we have $V_{n} z=z$. Furthermore, one has $\left(1-w_{n}\right) z+w_{n} U_{n} z=z$. It is easy to get $U_{n} z=z$. Thus, we find from (3.2) and (3.6) that

$$
\begin{aligned}
& \left\|x_{n+1}-z\right\|^{2} \\
& =\left\|\alpha_{n} h\left(x_{n}\right)+\left(1-\alpha_{n}\right)\left(V_{n} x_{n}+\tilde{e}_{n}\right)-z\right\|^{2} \\
& \leq\left\|\alpha_{n} h\left(x_{n}\right)+\left(1-\alpha_{n}\right) V_{n} x_{n}-z\right\|^{2}+M_{2}\left\|\tilde{e}_{n}\right\| \\
& =\left\|V_{n} x_{n}-z+\alpha_{n}\left(h\left(x_{n}\right)-V_{n} x_{n}\right)\right\|^{2}+M_{2}\left\|\tilde{e}_{n}\right\| \\
& =\left\|V_{n} x_{n}-z\right\|^{2}+\alpha_{n}{ }^{2}\left\|h\left(x_{n}\right)-V_{n} x_{n}\right\|^{2}+2 \alpha_{n}\left\langle V_{n} x_{n}-z, h\left(x_{n}\right)-V_{n} x_{n}\right\rangle+M_{2}\left\|\tilde{e}_{n}\right\| \\
& =\left\|\left(1-w_{n}\right) x_{n}+w_{n} U_{n} x_{n}-z\right\|^{2}+\alpha_{n}{ }^{2}\left\|h\left(x_{n}\right)-V_{n} x_{n}\right\|^{2} \\
& \quad+2 \alpha_{n}\left\langle V_{n} x_{n}-z, h\left(x_{n}\right)-V_{n} x_{n}\right\rangle+M_{2}\left\|\tilde{e}_{n}\right\| \\
& =\left(1-w_{n}\right)\left\|x_{n}-z\right\|^{2}+w_{n}\left\|U_{n} x_{n}-U_{n} z\right\|^{2}-w_{n}\left(1-w_{n}\right)\left\|U_{n} x_{n}-x_{n}\right\|^{2} \\
& \quad+\alpha_{n}{ }^{2}\left\|h\left(x_{n}\right)-V_{n} x_{n}\right\|^{2}+2 \alpha_{n}\left\langle V_{n} x_{n}-z, h\left(x_{n}\right)-V_{n} x_{n}\right\rangle+M_{2}\left\|\tilde{e}_{n}\right\| \\
& \leq\left\|x_{n}-z\right\|^{2}-w_{n}\left(1-w_{n}\right)\left\|U_{n} x_{n}-x_{n}\right\|^{2}+\alpha_{n}{ }^{2}\left\|h\left(x_{n}\right)-V_{n} x_{n}\right\|^{2} \\
& \quad+2 \alpha_{n}\left\langle V_{n} x_{n}-z, h\left(x_{n}\right)-V_{n} x_{n}\right\rangle+M_{2}\left\|\tilde{e}_{n}\right\| .
\end{aligned}
$$

Furthermore, we set

$$
\begin{aligned}
& s_{n}=\left\|x_{n}-z\right\|^{2}, \quad \gamma_{n}=\alpha_{n}\left(2-\alpha_{n}\left(1+2 \rho^{2}\right)-2\left(1-\alpha_{n}\right) \rho\right), \\
& \delta_{n}=\frac{1}{2-\alpha_{n}\left(1+2 \rho^{2}\right)-2\left(1-\alpha_{n}\right) \rho}\left[2 \alpha_{n}\|h(z)-z\|^{2}+M_{2} \frac{\left\|\tilde{e}_{n}\right\|}{\alpha_{n}}+2\left(1-\alpha_{n}\right)\left\langle h(z)-z, V_{n} x_{n}-z\right\rangle\right], \\
& \eta_{n}=w_{n}\left(1-w_{n}\right)\left\|U_{n} x_{n}-x_{n}\right\|^{2}, \text { and } \\
& \varphi_{n}=\alpha_{n}^{2}\left\|h\left(x_{n}\right)-V_{n} x_{n}\right\|^{2}+2 \alpha_{n}\left\langle V_{n} x_{n}-z, h\left(x_{n}\right)-V_{n} x_{n}\right\rangle+M_{2}\left\|\tilde{e}_{n}\right\| .
\end{aligned}
$$

Note that $\gamma_{n} \rightarrow 0, \Sigma_{n=0}^{\infty} \gamma_{n}=\infty\left(\lim _{n \rightarrow \infty}\left(2-\alpha_{n}\left(1+2 \rho^{2}\right)-2\left(1-\alpha_{n}\right) \rho\right)=2(1-\rho)>0\right)$ and $\varphi_{n} \rightarrow 0$ $\left(\alpha_{n} \rightarrow 0\right)$. From Lemma 2.9, it suffices to verify that $\eta_{n_{k}} \rightarrow 0(k \rightarrow \infty)$ implies that $\limsup _{k \rightarrow \infty} \delta_{n_{k}} \leq 0$ for any subsequence $\left(n_{k}\right) \subset(n)$. Indeed, $\eta_{n_{k}} \rightarrow 0(k \rightarrow \infty)$ implies that $\left\|U_{n_{k}} x_{n_{k}}-x_{n_{k}}\right\| \rightarrow 0(k \rightarrow \infty)$ due to condition (iii). It follows from (3.7) that

$$
\left\|x_{n_{k}}-V_{n_{k}} x_{n_{k}}\right\|=w_{n_{k}}\left\|x_{n_{k}}-U_{n_{k}} x_{n_{k}}\right\| \rightarrow 0 .
$$

Step 3. Show that

$$
\omega_{w}\left(x_{n_{k}}\right) \subset S
$$

where $\omega_{w}\left(x_{n_{k}}\right)$ is the set of all weak cluster points of $\left\{x_{n_{k}}\right\}$.

Take $\tilde{x} \in \omega_{w}\left\{x_{n_{k}}\right\}$ and assume that $\left\{x_{n_{k_{j}}}\right\}$ is a subsequence of $\left\{x_{n_{k}}\right\}$ weakly converging to $\tilde{x}$. Without loss of generality, we still use $\left\{x_{n_{k}}\right\}$ to denote $\left\{x_{n_{k_{j}}}\right\}$. Assume $\tau_{n_{k}} \rightarrow \tau$. Then $0<\tau<\frac{1}{L}$. Similarly, we take a subsequence $\left\{\lambda_{n_{k}}\right\}$ of $\left\{\lambda_{n}\right\}$ by condition (ii), and assume $\lambda_{n_{k}} \rightarrow \lambda$. Letting $V=J_{\lambda}^{B}\left(I-\tau A^{*}(I-\right.$ $T) A$ ), we see that $V$ is nonexpansive. Set

$$
t_{k}=x_{n_{k}}-\tau_{n_{k}} A^{*}(I-T) A x_{n_{k}}, \quad z_{k}=x_{n_{k}}-\tau A^{*}(I-T) A x_{n_{k}} .
$$


Using the resolvent identity, we deduce that

$$
\begin{aligned}
& \left\|V_{n_{k}} x_{n_{k}}-V x_{n_{k}}\right\| \\
& =\left\|J_{\lambda_{n_{k}}}^{B}\left(x_{n_{k}}-\tau_{n_{k}} A^{*}(I-T) A x_{n_{k}}\right)-J_{\lambda}^{B}\left(x_{n_{k}}-\tau A^{*}(I-T) A x_{n_{k}}\right)\right\| \\
& =\left\|J_{\lambda}^{B}\left(\frac{\lambda}{\lambda_{n_{k}}} t_{k}+\left(1-\frac{\lambda}{\lambda_{n_{k}}}\right) J_{\lambda_{n_{k}}}^{B} t_{k}\right)-J_{\lambda}^{B}\left(z_{k}\right)\right\| \\
& \leq\left\|\frac{\lambda}{\lambda_{n_{k}}} t_{k}+\left(1-\frac{\lambda}{\lambda_{n_{k}}}\right) J_{\lambda_{n_{k}}}^{B} t_{k}-z_{k}\right\| \\
& \leq \frac{\lambda}{\lambda_{n_{k}}}\left\|t_{k}-z_{k}\right\|+\left(1-\frac{\lambda}{\lambda_{n_{k}}}\right)\left\|J_{\lambda_{n_{k}}}^{B} t_{k}-z_{k}\right\| \\
& =\frac{\lambda}{\lambda_{n_{k}}}\left|\tau_{n_{k}}-\tau\right|\left\|A^{*}(I-T) A x_{n_{k}}\right\|+\left(1-\frac{\lambda}{\lambda_{n_{k}}}\right)\left\|J_{\lambda_{n_{k}}}^{B} t_{k}-z_{k}\right\| .
\end{aligned}
$$

Since $\lambda_{n_{k}} \rightarrow \lambda$ and $\tau_{n_{k}} \rightarrow \tau$ as $k \rightarrow \infty$, we immediately derive from the last relation that $\left\|V_{n_{k}} x_{n_{k}}-V x_{n_{k}}\right\| \rightarrow$ 0 . As a result, we find

$$
\left\|x_{n_{k}}-V x_{n_{k}}\right\| \leq\left\|x_{n_{k}}-V_{n_{k}} x_{n_{k}}\right\|+\left\|V_{n_{k}} x_{n_{k}}-V x_{n_{k}}\right\| \rightarrow 0 .
$$

Using Lemma 2.8, we get $\omega_{w}\left(x_{n_{k}}\right) \subset$ Fix $(V)$. It follows from Lemma 1.1 that $\omega_{w}\left(x_{n_{k}}\right) \subset S$. We also have

$$
\begin{aligned}
& \limsup _{k \rightarrow \infty}\left\langle h\left(x^{*}\right)-x^{*}, V_{n_{k}} x_{n_{k}}-x^{*}\right\rangle \\
= & \limsup _{k \rightarrow \infty}\left\langle h\left(x^{*}\right)-x^{*}, x_{n_{k}}-x^{*}\right\rangle+\lim _{k \rightarrow \infty}\left\langle h\left(x^{*}\right)-x^{*}, V_{n_{k}} x_{n_{k}}-x_{n_{k}}\right\rangle,
\end{aligned}
$$

and

$$
\limsup _{k \rightarrow \infty}\left\langle h\left(x^{*}\right)-x^{*}, x_{n_{k}}-x^{*}\right\rangle=\left\langle h\left(x^{*}\right)-x^{*}, \tilde{x}-x^{*}\right\rangle, \quad \forall \tilde{x} \in S .
$$

It is easy to get from (3.9) the second item of (3.13) tends to zero. Also, since $x^{*}$ is the unique solution of variational inequality problem (3.1), we obtain that

$$
\left\langle h\left(x^{*}\right)-x^{*}, \tilde{x}-x^{*}\right\rangle \leq 0 .
$$

Hence $\lim \sup _{k \rightarrow \infty} \delta_{n_{k}} \leq 0$.

The bounded perturbation of (3.4) by the following iterative process:

$$
\left\{\begin{array}{l}
y_{n}=x_{n}+\beta_{n} v_{n}, \\
x_{n+1}=\alpha_{n} h\left(y_{n}\right)+\left(1-\alpha_{n}\right) J_{\lambda_{n}}^{B}\left(\left(I-\tau_{n} A^{*}(I-T) A\right) y_{n}+e\left(y_{n}\right)\right) .
\end{array}\right.
$$

Similarly, we put

$$
\tilde{e}_{n}=J_{\lambda_{n}}^{B}\left(\left(I-\tau_{n} A^{*}(I-T) A\right) y_{n}+e\left(y_{n}\right)\right)-J_{\lambda_{n}}^{B}\left(I-\tau_{n} A^{*}(I-T) A\left(y_{n}\right)\right) .
$$

Theorem 3.2. Assume the sequences $\left\{\beta_{n}\right\}$ and $\left\{v_{n}\right\}$ satisfy condition (1.7). Let $H_{1}, H_{2}$ be two real Hilbert spaces and let $A$ be a bounded linear operator with $L=\left\|A^{*} A\right\|$, where $A^{*}$ is the adjoint of $A$. Suppose that $B: H_{1} \rightarrow 2^{H_{1}}$ is a maximal monotone operator and $T: H_{2} \rightarrow H_{2}$ is a nonexpansive mapping. Assume that $S=B^{-1} 0 \cap A^{-1} F i x(T) \neq \emptyset$. Let $h$ be a $\rho$-contractive mapping on $H_{1}$ with $0 \leq \rho<1$. Choose $x_{0} \in H_{1}$ arbitrarily and define the sequence $\left\{x_{n}\right\}$ by (3.15). If the following conditions are satisfied:

(i) $\lim _{n \rightarrow \infty} \alpha_{n}=0$ and $\sum_{n=0}^{\infty} \alpha_{n}=\infty$;

(ii) $0<\liminf _{n \rightarrow \infty} \lambda_{n} \leq \limsup \sup _{n \rightarrow \infty} \lambda_{n}<\infty$; 
(iii) $0<\liminf _{n \rightarrow \infty} \tau_{n} \leq \limsup _{n \rightarrow \infty} \tau_{n}<\frac{1}{L}$;

(iv) $\sum_{n=0}^{\infty}\left\|e\left(y_{n}\right)\right\|<\infty$,

then $\left\{x_{n}\right\}$ converges strongly to $x^{*}$, where $x^{*}$ is a solution of problem (1.5), which is also the unique solution of variational inequality problem (3.1).

Proof. We can rewrite (3.15) as

$$
x_{n+1}=\alpha_{n} h\left(x_{n}\right)+\left(1-\alpha_{n}\right)\left(J_{\lambda_{n}}^{B}\left(I-\tau_{n} A^{*}(I-T) A\right)\left(x_{n}\right)+\tilde{e}_{n}\right)+\bar{e}_{n},
$$

where

$$
\bar{e}_{n}=\alpha_{n}\left(h\left(y_{n}\right)-h\left(x_{n}\right)\right)+\left(1-\alpha_{n}\right)\left(J_{\lambda_{n}}^{B}\left(I-\tau_{n} A^{*}(I-T) A\right) y_{n}-J_{\lambda_{n}}^{B}\left(I-\tau_{n} A^{*}(I-T) A\right) x_{n}\right),
$$

In fact, by Proposition 2.7 (i), we see that $A^{*}(I-T) A$ is $\frac{1}{2 L}$-ism. It is not hard to see that it is $2 L$-Lipschitz. Thus,

$$
\begin{aligned}
\left\|\bar{e}_{n}\right\| & \leq \alpha_{n}\left\|h\left(y_{n}\right)-h\left(x_{n}\right)\right\|+\left(1-\alpha_{n}\right)\left\|y_{n}-x_{n}-\tau_{n}\left(A^{*}(I-T) A y_{n}-A^{*}(I-T) A x_{n}\right)\right\| \\
& \leq \alpha_{n} \rho\left\|y_{n}-x_{n}\right\|+\left(1-\alpha_{n}\right)\left(\left\|y_{n}-x_{n}\right\|+2 \tau_{n} L \| y_{n}-x_{n}\right) \| \\
& \leq\left(\alpha_{n} \rho+\left(1-\alpha_{n}\right)\left(1+2 \tau_{n} L\right)\right) \beta_{n}\left\|v_{n}\right\| .
\end{aligned}
$$

From condition (1.7), it turns out $\sum_{n=0}^{\infty}\left\|\bar{e}_{n}\right\|<\infty$. Consequently, we find from Theorem 3.1 that algorithm (3.4) is bounded perturbation resilient.

\section{NumericAl RESUltS}

In this section, we consider the following numerical example to demonstrate the effectiveness, realization, and convergence of Theorem 3.1.

Let $H_{1}=H_{2}=\mathbb{R}^{2}$. Define $h(x)=\frac{1}{10} x$. Take $B: \mathbb{R}^{2} \rightarrow \mathbb{R}^{2}$ and $T: \mathbb{R}^{2} \rightarrow \mathbb{R}^{2}$ as follows:

$$
B=\left(\begin{array}{ll}
8 & 0 \\
0 & 2
\end{array}\right)
$$

and

$$
T=\left(\begin{array}{cc}
\cos \theta & \sin \theta \\
-\sin \theta & \cos \theta
\end{array}\right) .
$$

Observe that $B$ is a positive linear operator. Then it is maximal monotone. $T$ is a rotation operator. Then it is nonexpansive. So, we obtain the resolvent mapping $J_{\lambda}^{B}=(I+\lambda B)^{-1}$. It follows that

$$
J_{\lambda}^{B}=\frac{1}{(8 \lambda+1)(2 \lambda+1)}\left(\begin{array}{cc}
2 \lambda+1 & 0 \\
0 & 8 \lambda+1
\end{array}\right) .
$$

Generate a $2 \times 2$ random matrix $A$, and compute the Lipschitz constant $L=\left\|A^{T} A\right\|$, where $A^{T}$ represents the transpose of $A$. Take $\lambda_{n}=0.5, \tau_{n}=\tau=\frac{1}{100 * L}$ and $\alpha_{n}=\frac{1}{n+1}$.

According to the iterative process of Theorem 3.1, the sequence $\left\{x_{n}\right\}$ is generated by

$$
x_{n+1}=\frac{1}{n+1} * \frac{1}{10} x_{n}+\left(1-\frac{1}{n+1}\right) J_{\lambda_{n}}^{B}\left(x_{n}-\tau_{n} A^{T}(I-T) A x_{n}\right) .
$$

As $n \rightarrow \infty$, we have $\left\{x_{n}\right\} \rightarrow x^{*}$. Taking random initial guess $x_{0}$, we obtain the numerical experiment results in Table 1 . 
TABLE $1 . x_{0}=\operatorname{rand}(2,1)$.

\begin{tabular}{ccccc}
\hline$\tau=\frac{1}{100 * L}$ & $\mathrm{n}$ (iterative number) & time $(s)$ & $x_{n}$ & $\operatorname{err}\left(\left\|x_{n+1}-x_{n}\right\|\right)$ \\
\hline 0.0069 & 20 & 0.000067 & $10^{-6} *[0.00000 .1210]^{T}$ & $6.2812 * 10^{-8}$ \\
\hline 0.0070 & 22 & 0.000073 & $10^{-7} *[-0.00020 .1638]^{T}$ & $8.4813 * 10^{-9}$ \\
\hline 0.0173 & 27 & 0.000087 & $10^{-9} *[-0.00020 .9495]^{T}$ & $4.8840 * 10^{-10}$ \\
\hline
\end{tabular}

Next, we consider the algorithm with bounded perturbation resilience. Choose the bounded sequence $\left\{v_{n}\right\}$ and the summable nonnegative real sequence $\left\{\beta_{n}\right\}$ as follows:

$$
v_{n}=\left\{\begin{array}{c}
-\frac{d_{n}}{\left\|d_{n}\right\|}, \quad \text { if } 0 \neq d_{n} \in B\left(x_{n}\right), \\
0, \quad \text { if } 0 \in B\left(x_{n}\right) .
\end{array}\right.
$$

where $B\left(x_{n}\right)=\left(8 x_{n}(1) 2 x_{n}(2)\right)^{T}, x_{n}(i), \quad i=1,2$ denotes the ith element of $x_{n}$, and $\beta_{n}=c^{n}$, for some $c \in(0,1)$. Setting $c=0.5$, the numerical results can be seen in Table 2 .

TABLE 2. $x_{0}=\operatorname{rand}(2,1)$.

\begin{tabular}{ccccc}
\hline$\tau=\frac{1}{100 * L}$ & $\mathrm{n}$ (iterative number) & time $(s)$ & $x_{n}$ & $\operatorname{err}\left(\left\|x_{n+1}-x_{n}\right\|\right)$ \\
\hline 0.0173 & 19 & 0.002022 & $10^{-6} *[0.68640 .0000]^{T}$ & $5.5268 * 10^{-7}$ \\
\hline 0.0056 & 22 & 0.000125 & $10^{-7} *[-0.85730 .0001]^{T}$ & $6.8973 * 10^{-8}$ \\
\hline 0.0046 & 25 & 0.002163 & $10^{-7} *[-0.10700 .0000]^{T}$ & $8.6055 * 10^{-9}$ \\
\hline
\end{tabular}

As we have seen, the error of the solution becomes smaller as the increasing iterative numbers. And, sequence $\left\{x_{n}\right\}$ converges to $(0,0)$ which is the solution of the example. Of course, it is also the unique solution of the variational inequality $\left\langle(I-h) x^{*}, x-x^{*}\right\rangle \geq 0$.

\section{Acknowledgements}

The first author was supported by the National Natural Science Foundation (No.11501566) and the Fundamental Research Funds for the Central Universities (No.3122017072).

\section{REFERENCES}

[1] Y. Censor, T. Elfving, A multiprojection algorithm using Bregman projections in product space, Numer. Algorithms, 8 (1994), 221-239.

[2] C. Byrne, Iterative oblique projection onto convex sets and the split feasibility problem, Inverse probl. 18 (2002), $441-453$.

[3] Y. Censor, A. Motova, A. Segal, Perturbed projections and subgradient projections for the multiple-sets split feasibility problem, J. Math. Anal. Appl. 327 (2007), 1244-1256.

[4] A. Moudafi, A note on the split common fixed-point problem for quasi-nonexpansive operators, Nonlinear Anal. 74 (2011), 4083-4087

[5] R. Kraikaew, S. Saejung, On split common fixed point problems, J. Math. Anal. Appl. 415 (2014), 513-524.

[6] W. Takahashi, H.K. Xu, J.C. Yao, Iterative methods for generalized split feasibility problems in Hilbert space, Set Valued Var. Anal. 23 (2015), 205-221.

[7] C. Byrne, Y. Censor, A. Gibali, S. Reich, Weak and strong convergence of algorithms for the split common null point problem, J. Nonlinear convex Anal. 13 (2012), 759-775. 
[8] P.L. Combettes, Solving monotone inclusions via compositions of nonexpansive averaged operators, Optimization 53 (2004), 475-504.

[9] X. Qin, A. Petrusel, J.C. Yao, CQ iterative algorithms for fixed points of nonexpansive mappings and split feasibility problems in Hilbet spaces, J. Nonlinear Convex Anal. 19 (2018), 157-165.

[10] Q.L. Dong, S. He, J. Zhao, Solving the split equality problem without prior knowledge of operator norms, Optimization, 64 (2015), 1887-1906.

[11] B. Qu, B. Liu, N. Zheng, On the computation of the step-size for the CQ-like algorithms for the split feasibility problem, Appl. Math. Comput. 262 (2015), 218-223.

[12] A. Moudafi, Split monotone variational inclusions, J. Optim. Theory Appl. 150 (2011), 275-283.

[13] S.Y. Cho, et al., Viscosity approximation splitting methods for monotone and nonexpansive operators in Hilbert spaces, J. Nonlinear Convex Anal. 19 (2018), 251-264.

[14] J. Zhao, Y. Zhang, Q. Yang, Modified projection methods for the split feasibility problem and the multiple-sets split feasibility problem, Appl. Math. Comput. 219 (2012), 1644-1653.

[15] X. Qin, J.C. Yao, Projection splitting algorithms for nonself operators, J. Nonlinear Convex Anal. 18 (2017), 925-935.

[16] Y. Censor, R. Davidi, G.T. Herman, Perturbation resilience and superiorization of iterative algorithms, Inverse Probl. 26 (2010), Article ID 65008.

[17] Y. Censor, A.J. Zaslavski, Convergence and perturbation resilience of dynamic string-averaging projection methods, Comput. Optim. Appl. 54 (2013), 65-76.

[18] Q.L. Dong, J. Zhao, S. He, Bounded perturbation resilience of the viscosity algorithm, J. Inequal. Appl. 299 (2016), 1-12.

[19] W. Jin, Y. Censor, M. Jiang, Bounded perturbation resilience of projected scaled gradient methods, Comput. Optim. Appl. 63 (2016), 365-392.

[20] H.K. Xu, Bounded perturbation resilience and superiorization techniques for the projected scaled gradient method, Inverse Probl. 33 (2017), Article ID 044008.

[21] Y. Censor, W. Chen, P.L. Combettes, R. Davidi, G.T. Herman, On the effectiveness of projection methods for convex feasibility problem with linear inequality constrains, Comput. Optim. Appl. 51 (2012), 1065-1088.

[22] R. Davidi, Y. Censor, R.W. Schulte, S. Geneser, L. Xing, Feasibility-seeking and superiorization algorithm applied to inverse treatment plannning in rediation therapy, Contemp. Math. 636 (2015), 83-92.

[23] R. Davidi, G.T. Herman, Y. Censor, Perturbation-resilient block-iterative projection methods with application to image reconstruction from projections, Int. Trans. Oper. Res. 16 (2009), 505-524.

[24] Y.N. Guo, W. Cui, Strong convergence and bounded perturbation resilience of a modified proximal gradient algorithm, J. Inequal. Appl. 2018 (2018), Article ID 103.

[25] H.K. Xu, averaged mappings and the gradient-projection algorithm, J. Optim. Theory Appl. 150 (2011), 360-378.

[26] K. Geobel, W.A. Kirk, Topics in metric fixed point theory, Cambridge Studies in Advanced Mathematics, Cambridge University Press, Cambridge, 1990.

[27] C. Yang, S. He, General alterative regularization methods for nonexpansive mappings in Hilbert spaces, Fixed Point Theory Appl. 2014 (2014), Article ID 203.

[28] V. Barbu, Nonlinear Semigroups and Differential Equations in Banach Spaces, Noordhoff, Amsterdam, 1976.

[29] A. Moudafi, Viscosity approximation methods for fixed point problems, J. Math. Anal. Appl. 241 (2000), $46-55$.

[30] X. Qin, S.Y. Cho, L. Wang, Iterative algorithms with errors for zero points of m-accretive operators, Fixed Point Theory Appl. 2013 (2013), Article ID 148.

[31] J.S. Jung, Viscosity approximation methods for a family of finite nonexpansive mappings in Banach spaces, Nonlinear Anal. 64 (2006), 2536-2552.

[32] S.S. Chang, Viscosity approximation methods for a finite family of nonexpansive mappings in Banach spaces, J. Math. Anal. Appl. 323 (2006) 1402-1416. 